\title{
Desenvolvimento de um Chatbot como Ferramenta de Informação para Usuários em Geral sobre a COVID-19
}

\author{
Marllon Sóstenes dos Santos Brito ${ }^{1}$, Fábio José Gomes de Sousa ${ }^{1}$, Raquel Silveira ${ }^{1}$, \\ Emanoel Avelar Muniz ${ }^{1}$
}

${ }^{1}$ Instituto Federal de Educação, Ciência e Tecnologia do Ceará (IFCE)

marllonsostenes@gmail.com, ffabio.jose, raquel_silveira, emanoel.muniz\}@ifce.edu.br

\begin{abstract}
This meta-paper describes the development of a mobile application to disseminate official information about COVID-19 to users in general through a chat agent (COVID-BOT), using DialogFlow library. The purpose of this application is to facilitate access to information about COVID-19, answering general questions from users, providing guidance on the vaccination plan and coronavirus test, thus helping to disseminate true information and reduce misinformation.
\end{abstract}

Resumo. Este artigo propõe o desenvolvimento de um aplicativo móvel para divulgação de informações oficiais sobre a COVID-19 para usuários em geral, por meio de um agente de conversação (COVID-BOT), utilizando a biblioteca DialogFlow. O objetivo desta aplicação é facilitar o acesso à informação sobre a COVID-19, respondendo dúvidas gerais dos usuários e fornecendo orientações sobre o plano de vacinação e os testes de coronavírus, ajudando a disseminar informações verdadeiras e diminuir a desinformação.

\section{Introdução}

A preocupação com a saúde mental da população se intensifica durante uma grave crise social. Estamos vivendo a pandemia do novo coronavírus desde o dia 11 de março de 2020. Inicialmente, em 31 de janeiro de 2020, a Organização Mundial de Saúde (OMS) declarou a infecção pelo novo coronavírus como emergência global e a seguir nomeou a doença de COVID-19. A COVID-19, nome da síndrome respiratória ocasionada pelo novo coronavírus, foi inicialmente detectada em 2019 na cidade de Wuhan, capital da província da China Central. Ela atingiu as pessoas em diferentes níveis de complexidade, sendo os casos mais graves acometidos de uma insuficiência respiratória aguda que requer cuidados hospitalares intensivos - incluindo o uso de ventilação mecânica [Centers for Disease Control and Prevention 2020].

O Grupo de Estudos de Coronavírus do Comitê Internacional de Taxonomia de Vírus propôs que o vírus seja designado de SARS-Cov-2 [Ruan e Yang 2020]. O quadro clínico da COVID-19 é semelhante ao de outras viroses respiratórias, como febre, tosse geralmente seca, cansaço e, em casos mais graves (5\%), dispneia, sangramento pulmonar, linfopenia grave e insuficiência renal. Em $80 \%$ dos casos, os sintomas são leves. O diagnóstico dos casos sintomáticos deve ser confirmado com a pesquisa do vírus por reação em cadeia da polimerase (PCR) de swab nasal ou pela coleta de sangue sendo analisada a presença ou ausência do vírus. Até meados de abril de 2020 haviam sido contabilizados mais de dois milhões de casos notificados e quase 150 mil mortes no mundo, com os Estados Unidos (EUA) liderando a quantidade de óbitos (mais de 25 mil). De acordo com o Center for Systems Science and Engineering (CSSE), na 
Universidade de Johns Hopkins, o total de casos para o Brasil em 25 de março de 2021 é de 12.220.011 pessoas, com 10.750.131 pessoas recuperadas e 300.685 mortes pelo vírus, distribuídos em 89.992 casos por dia. No Ceará, em 25 de março de 2021 o total de casos é de 516.556 pessoas infectadas e 13.313 mortes pelo vírus [Dong 2021].

Os centros de pesquisas vêm cumprindo o seu papel de combate à crise global, redobrando seus esforços de pesquisa e desenvolvimento para encontrar tratamentos, desenvolver e produzir vacinas, insumos e equipamentos para auxiliar a área da saúde. A facilidade de propagação, a falta de conhecimento sobre o vírus e o aumento exponencial do número de contágios fizeram com que a OMS elevasse a doença ao status de pandemia em março de 2020 [WHO 2020].

A pandemia do novo Coronavírus trouxe também outra pandemia: a de notícias falsas e de desinformação. Enquanto as autoridades médicas e sanitárias do país e do mundo recomendam o isolamento e alertam contra o uso de medicamentos sem validação científica, grupos mal intencionados espalham notícias falsas, através das redes sociais e em grupos de mensagens em massa, ora desprezando a dimensão da crise, ora incitando a população a abandonar as medidas necessárias, gerando um transtorno à saúde pública [DE SOUSA JÚNIOR 2020].

Diante disso, há necessidade de mecanismos efetivos que permitam disseminar o acesso à informação verdadeira e diminuir a desinformação sobre a COVID-19. Além dos meios de comunicação oficiais de imprensa, há um assistente virtual que apresenta informações oficiais, no site do Governo do Estado do Ceará, sobre a pandemia, porém ainda não suficientes [SESA 2021]. Ressalta-se que a grande maioria da população brasileira dispõe de celular com sistema Android que lhe permite receber essas informações por outras vias. Neste contexto, este trabalho propõe o desenvolvimento de um aplicativo chatbot, que usa a biblioteca DialogFlow funcional em dispositivos Android, que implementa os cenários sobre: informações gerais, testes e plano de vacinação sobre a COVID-19, tendo como base documentos oficiais da Secretaria da Saúde do Estado do Ceará (SESA), visando contribuir com a informação oficial e o combate às fake news.

\section{Método e Desenvolvimento do Chatbot}

Diante da necessidade de disponibilização de canais para levar informações às pessoas acerca da COVID-19, este trabalho propõe o desenvolvimento do COVID-BOT, um robô de conversa com usuários em geral, capaz de apresentar informações concisas de fontes confiáveis e mais rápidas do que a longa lista de resultados de mídia social ou mecanismos de pesquisa na web.

A Figura 1 apresenta o fluxo de desenvolvimento do COVID-BOT, composto por 4 (quatro) etapas principais. Cada etapa é detalhada a seguir. As etapas 1 e 2, Elaboração dos cenários e Desenvolvimento do chatbot, respectivamente, foram desenvolvidas neste trabalho, enquanto que as etapas 3 e 4, Experimentação e Disponibilização, respectivamente, serão desenvolvidas em trabalhos futuros. 


\subsection{Elaboração dos cenários}

Inicialmente, foi realizada uma pesquisa para coleta de material e informações para o conteúdo do chatbot. O conteúdo investigado precisou ser analisado com cautela, pois as informações usadas exigiam ser, sobretudo, seguras, precisas e atualizadas. À vista disso, optou-se por utilizar os seguintes documentos: (i) Plano Estadual de Contingência para Resposta às Emergências em Saúde Pública Doença pelo Coronavírus 2019 (COVID-19) [SESA 2021b], (ii) Perguntas e Respostas com Esclarecimentos Básicos sobre Testes para Detecção do Novo Coronavírus (Sars-CoV-2) em Serviços de Saúde [ANVISA 2021], e (iii) Plano de Operacionalização para Vacinação contra COVID-19 [SESA 2021a].

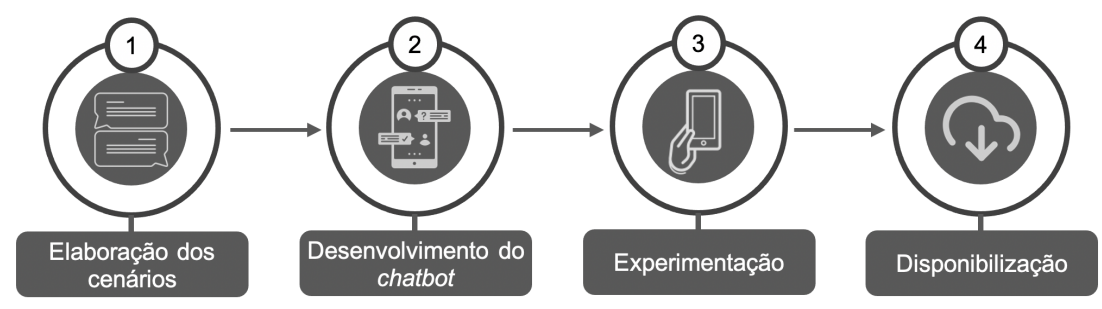

Figura 1. Etapas de execução do COVID-BOT.

A partir desses documentos, foram elaboradas, pelos autores deste trabalho, situações de diálogos que visam orientar o usuário acerca da COVID-19 nas seguintes temáticas: (i) informações gerais (aborda, de forma geral, a origem, os principais sintomas, os grupos de risco e as medidas preventivas), (ii) testes (envolvem os tipos de exames que detectam se a pessoa teve ou não contato com o vírus) e (iii) plano de vacinação (contempla, de forma geral, as fases do plano de vacinação do estado do Ceará). Esses diálogos foram revisados por um profissional de saúde e incluídos em uma base de conhecimento de cenários de conversação, utilizada para identificar a resposta adequada durante a conversação do usuário com o chatbot.

\subsection{Desenvolvimento do chatbot}

O agente conversacional proposto neste trabalho é aplicado a dispositivos móveis com sistema operacional Android. A interface do chatbot foi dividida no desenvolvimento do front-end (apresentação do software ao usuário) e do back-end (componente que fornece respostas ao usuário).

$\mathrm{O}$ desenvolvimento do front-end tem o intuito de facilitar o entendimento dos requisitos do chatbot, de apresentar para o usuário as funcionalidades do software de modo simplificado e de aumentar a percepção de valor. Para o desenvolvimento do front-end do COVID-BOT, inicialmente foi desenvolvida a prototipação em duas etapas: (i) prototipação de baixo nível (desenho das páginas da aplicação em lápis e papel) e (ii) protótipo de alta fidelidade (utilizando um Integrated Development Environment (IDE) e seguindo o protótipo de baixo nível, apresentando as principais páginas e interações). A prototipação de alto nível e a implementação das funcionalidades do front-end foram realizadas utilizando o Android Studio ${ }^{1}$, o IDE oficial para o desenvolvimento de

\footnotetext{
${ }^{1}$ https://developer.android.com/studio
} 
aplicativos Android, com a linguagem de programação Kotlin, juntamente com as bibliotecas do Android SDK na versão 30.1.1.

O back-end do COVID-BOT foi desenvolvido utilizando o DialogFlow ${ }^{2}$, uma API para a construção e integração de agentes conversacionais. Mais especificamente, o DialogFlow permite o armazenamento da base de conhecimento de cenários de conversação e faz uso de técnicas de inteligência artificial, aprendizagem de máquina e processamento de linguagem de máquina para interpretar a mensagem do usuário e gerar a resposta adequada a ser emitida pelo chatbot.

A Figura 2 apresenta uma visão geral da arquitetura do COVID-BOT, ilustrando como ocorre a comunicação do usuário com o chatbot. Inicialmente, a partir do front-end do COVID-BOT, o usuário envia uma mensagem (1), por exemplo, "Como me prevenir da COVID-19?". Essa mensagem é encaminhada para o DialogFlow (2) que é responsável por gerenciar o funcionamento do chatbot, ou seja, processa a mensagem do usuário e seleciona a resposta adequada na base de conhecimento de cenários de conversação para ser emitida pelo chatbot (3). Caso o chatbot não reconheça a mensagem do usuário, o assistente conversacional (COVID-BOT) emitirá uma resposta padrão ao usuário, como, por exemplo, “Desculpe-me, não entendi!". A resposta selecionada pelo DialogFlow é enviada para o front-end para leitura pelo usuário.

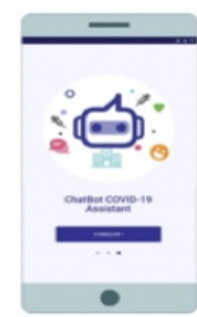

(1)

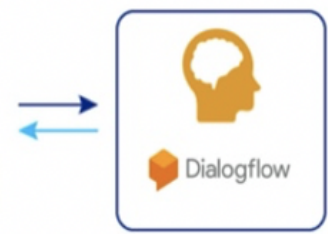

(2)

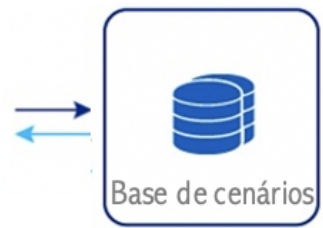

(3)

Figura 2. Visão geral da arquitetura do COVID-BOT

\subsection{Experimentação e Disponibilização}

Serão realizados experimentos empíricos no COVID-BOT com o intuito de avaliar a qualidade das informações fornecidas, demonstrando a aplicabilidade e viabilidade da abordagem proposta. Posteriormente, o COVID-BOT será disponibilizado na loja de aplicativos para uso pelos usuários em geral.

\section{Discussão}

A priori, as contribuições deste trabalho estão pautadas em dois aspectos: (i) no desenvolvimento inicial de uma base de conhecimento de cenários no contexto da COVID-19, envolvendo orientações para os usuários quanto às informações gerais sobre a COVID-19, testes e o plano de vacinação do estado do Ceará, além do (ii) desenvolvimento de um agente conversacional que interage com o usuário por meio de diálogos textuais considerando o contexto da base de conhecimento de cenários elaborada neste trabalho.

\footnotetext{
${ }^{2}$ https://dialogflow.cloud.google.com/
} 
A Tabela 2 apresenta a quantidade de diálogos conversacionais (perguntas e respostas) contidos, até o momento, na base de conhecimento de cenário e validados por um profissional da saúde. Os cenários de conversação relacionados às informações gerais sobre a COVID-19 abrangem orientações sobre a origem da COVID-19, prevenção, transmissão, sintomas, fatores de risco e tratamento. Os diálogos de conversação sobre testes de COVID-19 envolvem os tipos de testes. Enquanto que os cenários de conversação sobre o plano de vacinação abordam orientações sobre as vacinas, o cronograma de vacinação do estado do Ceará e os grupos prioritários.

Tabela 2. Quantidade de perguntas e respostas para cada temática de conversação.

\begin{tabular}{|l|c|}
\hline \multicolumn{1}{|c|}{ Temática dos cenários } & Quantidade de perguntas e respostas \\
\hline Informações gerais sobre a COVID-19 & 12 \\
\hline Testes & 10 \\
\hline Plano de vacinação & 18 \\
\hline
\end{tabular}

A Figura 3 ilustra, de forma simplificada, as páginas de conversação do COVID-BOT, após o processo de prototipação de baixa e alta fidelidade, considerando a interação do chatbot com o usuário nas seguintes temáticas presentes na base de conhecimento de cenários: (a) informações gerais e (b) vacinação. Por questão de espaço apenas alguns cenários e exemplos de trechos de conversação com o COVID-BOT são apresentados. Destaca-se que foi adotado o mesmo padrão de interface (ou seja, cores, navegabilidade, simplicidade e usabilidade) em todas as conversações.

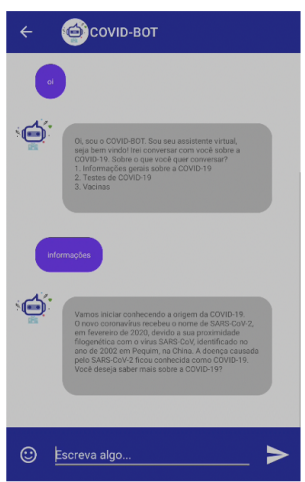

(a)

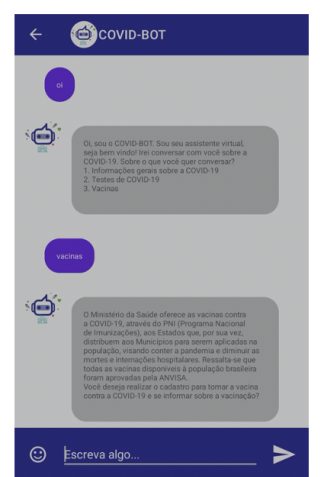

(b)

Figura 3. Páginas de interação do COVID-BOT com o usuário nas temáticas (a) informações gerais e (b) plano de vacinação.

\section{Considerações Finais}

A adição de uma plataforma conversacional sobre a COVID-19 entre os meios de divulgação da informação já existentes agregará direta e indiretamente na disseminação de informações corretas de como lidar no cotidiano com o coronavírus. Este trabalho apresenta o COVID-BOT, um agente conversacional criado para fornecer orientações aos usuários relacionadas à atual pandemia da COVID-19, trazendo respostas sobre informações gerais e testes da COVID-19, assim como o plano de vacinação do estado do Ceará. O chatbot proposto neste trabalho está em andamento, mas já apresenta um 
grande potencial de contribuição em fornecer orientações para os usuários e combater a desinformação relacionada à pandemia.

Como trabalhos futuros pretende-se ampliar a qualidade da base de conhecimento de cenários, adicionando novas temáticas e novos cenários de conversação, assim como realizar uma experimentação do COVID-BOT com usuários em geral e disponibilizar o chatbot na loja de aplicativos para uso de usuários em geral.

\section{Referências}

Agência Nacional de Vigilância Sanitária (ANVISA) (2021), "Perguntas e Respostas com Esclarecimentos Básicos sobre Testes para Detecção do Novo Coronavírus (Sars-CoV-2) em Serviços de Saúde". Disponível em: https://www.gov.br/anvisa/pt-br/centraisdeconteudo/publicacoes/produtos-para-a-sau de/manuais/perguntas-e-respostas-testes-para-covid-08-05-2020.pdf. Acesso em: 19 abr 2021.

Centers for Disease Control and Prevention (2020), "Severe Outcomes Among Patients with Coronavirus Disease 2019 (COVID-19)" DOI: http://dx.doi.org/10.15585/mmwr.mm6912e2.

DE SOUSA JÚNIOR, João Henriques et al. Da Desinformação ao Caos: uma análise das Fake News frente à pandemia do Coronavírus (COVID-19) no Brasil. Cadernos de Prospecção, v. 13, n. 2 COVID-19, p. 331, 2020.

Dong E, Du H, Gardner L. (2019), "An interactive web-based dashboard to track COVID-19 in real time. Lancet Infect Dis" Disponível em: https://www.arcgis.com/apps/opsdashboard/index.html\#/bda7594740fd40299423467 b48e9ecf6 Acesso em 25 mar. 2021.

Ruan Q, Yang K, Wang W, Jiang L, Song J. (2020), "Clinical predictors of mortality due to COVID-19 based on an analysis of data of 150 patients from Wuhan, China." Intensive Care Medicine. DOI: https://doi.org/10.1007/s00134-020-05991-X

Secretaria da Saúde do Estado do Ceará (SESA), Plantão Coronavírus (2020), canal whatsapp e assistente virtual Plantão Coronavírus disponível em: https://www.saude.ce.gov.br/2020/04/13/plantao-coronavirus-governo-do-ceara-lanca -canal-de-whatsapp-para-atender-a-populacao/. Acesso em 24 mai. 2021.

Secretaria da Saúde do Estado do Ceará (SESA), Secretaria Executiva de Vigilância em Saúde e Regulação. Coordenadoria de Vigilância Epidemiológica e Prevenção em Saúde (2021), Plano de Operacionalização para Vacinação contra COVID-19. versão 6, Ceará.

Secretaria da Saúde do Estado do Ceará (SESA), Secretaria Executiva de Vigilância em Saúde e Regulação. Coordenadoria de Vigilância Epidemiológica e Prevenção em Saúde (2021), Plano Estadual de Contingência para Respostas às Emergências em Saúde Pública doença pelo coronavírus 2019 (COVID-19). 6a edição, Ceará.

World Health Organization (WHO) (2019), "Coronavirus disease 2019 (COVID-19) Situation Report - 78." Disponível em: http://www.who.int/docs/default-source/coronaviruse/situation-reports/20200407-sitr ep-78-covid 19.pdf?sfvrsn=bc43e1b_2. Acesso em: 18 fev. 2021. 